Artículo

\title{
Definición de curvas de crecimiento con modelos no lineales en borregas de siete razas con registro de pureza en México
}

Joel Domínguez-Viveros ${ }^{\mathrm{a}^{*}}$

Edwin Canul-Santos ${ }^{a}$

Felipe Alonso Rodríguez-Almeida ${ }^{a}$

María Eduviges Burrola-Barraza ${ }^{\text {a }}$

Juan Ángel Ortega-Gutiérrez ${ }^{\text {a }}$

Francisco Castillo-Rangel ${ }^{a}$

${ }^{a}$ Universidad Autónoma de Chihuahua. Facultad de Zootecnia y Ecología. Periférico Francisco R. Almada km 1. 31453 Chihuahua, Chih. México.

* Autor de correspondencia: joeldguezviveros@yahoo.com.mx - jodominguez@uach.mx

\section{Resumen:}

Caracterizar el crecimiento ayuda en la toma de decisiones de manejo, comercialización y mejoramiento genético. El objetivo fue identificar un modelo no lineal (MNL) para describir la curva de crecimiento en borregas de registro a través de siete razas. Se evaluó el peso vivo, desde el nacimiento hasta los $230 \mathrm{~d}$ de edad, de las razas Blackbelly (BB; n= 19,084), Pelibuey (PE; $\mathrm{n}=$ 39,025), Dorper (DR; n= 35,814), Katahdin (KT; n= 74,154), Suffolk (SF; n= 10,267), Hampshire (HS; n= 7561) y Rambouillet (RB; n= 7,384). Se evaluaron los MNL: Brody (BRO), Verhulst (VER), Von Bertalanffy (VBE), Gompertz (GOM), Mitscherlich (MIT) y Logístico (LOG). Los análisis se realizaron con el software SAS. Los criterios para seleccionar el modelo con mejor ajuste fueron: error de predicción promedio, varianza del error de predicción, estadístico DurbinWatson, coeficiente de determinación, raíz del cuadrado medio del error, criterios de información Akaike y Bayesiano. Para HS, PE y SF, el mejor modelo fue VBE, con una curva sigmoide y edad al punto de inflexión entre 40 y 57 d. Los modelos BRO y MIT tuvieron el mejor ajuste para KT, $\mathrm{BB}, \mathrm{DR}$ y RB, con una curva continua, sin punto de inflexión y tasa de crecimiento constante. Para peso adulto se observaron marcadas diferencias, con valores promedio $(\mathrm{kg})$ de 44.6 en $\mathrm{BB}, 49.2$ en 
RB, 52.9 en PE, 55.6 en HS, 60.2 en KT, 64.7 en SF y 65.2 en DR; con la tendencia de valores mayores para los modelos BRO y MIT, y los menores para LOG y VER.

Palabras clave: Tasa de crecimiento, Peso adulto, Selección de modelos, Von Bertalanffy, Brody, Regresión no lineal.

Recibido: 10/03/2018

Aceptado: 27/09/2018

\section{Introducción}

El Organismo de la Unidad Nacional de Ovinocultores (OUNO) agrupa a los criadores de ovinos especializados y de registro de México. El OUNO coordina los esquemas de mejoramiento genético de las razas ovinas, con base en los registros genealógicos y los controles de producción de las variables incluidas en los criterios y objetivos de selección de cada raza. Con relación a las variables de crecimiento, se registra el peso vivo del animal en cinco puntos o edades ${ }^{(1)}$; los datos de peso vivo a diferentes edades generan una distribución de puntos a través del tiempo, que permite analizar y caracterizar el patrón de crecimiento del ovino con base en modelos matemáticos no lineales (MNL), los cuales resumen la variación del peso vivo a través del tiempo en un reducido número de parámetros e indicadores de crecimiento con interpretación biológica ${ }^{(2,3)}$.

La producción de ovinos en México se desarrolla en condiciones diversas de tecnología, agroecología y socioeconomía. El registro ordenado y verídico de los eventos que ocurren en la unidad de producción, particularmente de las características de interés económico, es fundamental para que el criador determine la rentabilidad de la unidad. El cambio del peso vivo del animal está influido por factores genéticos y ambientales, con efectos variables a través del tiempo o del desarrollo del individuo; por consiguiente, cada raza tiene un patrón de crecimiento con características propias, por lo que se requiere probar varios MNL para identificar el de mejor ajuste en cada raza. La identificación de modelos con el mejor ajuste proporciona información objetiva y precisa del patrón de crecimiento, que puede utilizarse por los criadores en la toma de decisiones relacionadas con producción, manejo y mejora genética.

Con base en lo anterior, los objetivos del presente estudio fueron: 1) identificar el MNL de mejor ajuste para describir la curva de crecimiento en ovinos de cuatro razas de pelo (Blackbelly, Pelibuey, Dorper y Katahdin) y tres razas de lana (Suffolk, Hampshire y Rambouillet); y, 2) generar indicadores de crecimiento que permitan caracterizar y analizar las curvas de crecimiento. 


\section{Material y métodos}

La base de datos analizada incluyó registros de peso vivo de borregas en siete razas de registro del OUNO: Blackbelly (BB), Pelibuey (PE), Dorper (DR), Katahdin (KT), Suffolk (SF), Hampshire (HS) y Rambouillet (RB). Las variables analizadas fueron los pesos vivos tomados al nacer, a los $75,120,150$ y 210 días de edad, con mediciones en intervalos de \pm 20 días con respecto a la edad de referencia (Cuadro 1). El peso a los 75 días corresponde al destete; dado las características del mercado los machos son comercializados a partir de los 120 días, por lo cual el presente estudio incluyó sólo datos de hembras.

Cuadro 1: Número de registros a diferentes edades en las siete razas de ovinos evaluadas

\begin{tabular}{lrrrrrr}
\hline Raza & PN & \multicolumn{1}{c}{ P75 } & P120 & \multicolumn{1}{c}{ P150 } & P210 & \multicolumn{1}{c}{ Total } \\
\hline Katahdin & 24,878 & 21,365 & 11,500 & 10,502 & 5,909 & 74,154 \\
Pelibuey & 14,164 & 11,796 & 5,301 & 4,993 & 2,771 & 39,025 \\
Dorper & 11,487 & 9,522 & 5,802 & 5,510 & 3,493 & 35,814 \\
Blackbelly & 7,151 & 5,439 & 2,475 & 2,416 & 1,603 & 19,084 \\
Suffolk & 3,636 & 2,836 & 1,542 & 1,459 & 794 & 10,267 \\
Hampshire & 2,597 & 2,177 & 1,236 & 1,056 & 495 & 7,561 \\
Rambouillet & 2,504 & 1,748 & 1,189 & 1,093 & 850 & 7,384 \\
\hline
\end{tabular}

$\mathrm{PN}=$ peso nacer; P75= peso vivo en el intervalo de 55 a $95 \mathrm{~d} ; \mathrm{P} 120=$ peso vivo en el intervalo de 100 a $140 \mathrm{~d}$; $\mathrm{P} 150=$ peso vivo en el intervalo de 130 a $170 \mathrm{~d} ; \mathrm{P} 210=$ peso vivo en el intervalo de 190 a $230 \mathrm{~d}$.

La información procedió de rebaños distribuidos en tres regiones del país, principalmente. En la zona centro se encuentra el $50 \%$ de los rebaños evaluados, produciendo ovinos de las razas SF, HS, RB, especialmente. De la región sur - sureste se derivó el $22 \%$ de la base de datos, correspondientes a las razas PE, BB, DR y KT; en la zona norte están ubicados el $18 \%$ de los rebaños, criando ovinos de las razas BB, DR y KT, principalmente; el $10 \%$ restante, procedió de rebaños de otras regiones del país. La zona centro se caracteriza por sistemas de producción intensivos o semi intensivos, en régimen de estabulación combinando pastoreo tecnificado. Las regiones norte y sur - sureste se caracterizan por sistemas de producción semi intensivos y extensivos, combinando los regímenes de pastoreo y confinamiento en corrales. En el norte se cuenta con grandes extensiones áridas y semiáridas donde se aprovechan pastizales y matorrales de diversas especies; en el sur - sureste con climas tropicales permite una alta disponibilidad de pastos tropicales.

Los modelos no lineales (MNL) que se evaluaron fueron: Brody (BRO), Verhulst (VER), Von Bertalanffy (VBE), Gompertz (GOM), Mitscherlich (MIT) y logístico (LOG). Todos ellos conformados por tres coeficientes $\left(\beta_{1}, \beta_{2}\right.$ y $\left.\beta_{3}\right)$ de regresión ${ }^{(4,5,6)}$. En las ecuaciones de los MNL 
(Cuadro 2), $\boldsymbol{y}_{\boldsymbol{i}}$ representa el peso vivo ( $\mathrm{kg}$ ) medido al tiempo $\boldsymbol{t}$; $\beta_{1}$, es el valor asintótico cuando $\boldsymbol{t}$ tiende a infinito, interpretado como el parámetro de peso adulto (PAD); $\beta_{2}$, es un parámetro de ajuste cuando $\boldsymbol{y} \neq 0$ y $\boldsymbol{t} \neq 0$; y $\beta_{3}$, es la tasa de crecimiento (TAC), expresando la ganancia de peso como proporción del peso total ${ }^{(2,7)}$. Los modelos VER, VBE, GOM y LOG se caracterizan por describir el crecimiento con base en una curva sigmoide, para los cuales se estimó la edad (EPI) y el peso (PPI) al punto de inflexión ${ }^{(8,9)}$.

Cuadro 2: Modelos no lineales utilizados para describir el crecimiento en ovinos de registro

\begin{tabular}{ll}
\hline Modelo & Ecuación \\
\hline Verhulst & $\mathrm{y}_{\mathrm{i}}=\beta_{1} *\left(1+\exp \left(-\beta_{2} * \mathrm{t}\right)\right)^{-\beta 3}+\mathrm{e}_{\mathrm{i}}$ \\
Logístico & $\mathrm{y}_{\mathrm{i}}=\beta_{1} /\left(1+\beta_{2} *\left(\exp \left(-\beta_{3} * \mathrm{t}\right)\right)\right)+\mathrm{e}_{\mathrm{i}}$ \\
Von Bertalanffy & $\left.\mathrm{y}_{\mathrm{i}}=\beta_{1} *\left(\left(1-\beta_{2} *\left(\exp \left(-\beta_{3} * \mathrm{t}\right)\right)\right)\right)^{* * 3}\right)+\mathrm{e}_{\mathrm{i}}$ \\
Gompertz & $\mathrm{y}_{\mathrm{i}}=\beta_{1} *\left(\exp \left(-\beta_{2} *\left(\exp \left(-\beta_{3} * \mathrm{t}\right)\right)\right)\right)+\mathrm{e}_{\mathrm{i}}$ \\
Brody & $\mathrm{y}_{\mathrm{i}}=\beta_{1} *\left(1-\beta_{2} *\left(\exp \left(-\beta_{3} * \mathrm{t}\right)\right)\right)+\mathrm{e}_{\mathrm{i}}$ \\
Mitscherlich & $\mathrm{y}_{\mathrm{i}}=\beta_{1} *\left(1-\exp \left(\beta_{3} * \beta_{2}-\beta_{3} * \mathrm{t}\right)\right)+\mathrm{e}_{\mathrm{i}}$ \\
\hline
\end{tabular}

$\mathrm{y}_{\mathrm{i}}=$ peso vivo en kg medido al $\mathrm{t}$ tiempo; $\beta_{1}=$ valor asintótico; $\beta_{2}=$ constante de integración; $\beta_{3}=$ pendiente de la curva o tasa de crecimiento.

Los análisis se realizaron con el método de Gauss-Newton del procedimiento NLIN del programa para análisis estadístico $\mathrm{SAS}^{(10)}$; la selección del modelo con mejor ajuste se realizó en función $\mathrm{de}^{(11,12,13)}$ : a) criterio de información Akaike $[\mathrm{AIC}=\mathrm{n} * \ln (\mathrm{sce} / \mathrm{n})+2 \mathrm{k}$; b) criterio de información Bayesiano $\left[\mathrm{BIC}=\mathrm{n}^{*} \ln (\mathrm{sce} / \mathrm{n})+\mathrm{k}^{*} \ln (\mathrm{n})\right]$; c) error de predicción promedio $[\mathrm{EPP}=$ $\left.\left.\left(\sum_{\mathrm{i}=1}^{\mathrm{n}}\left(\frac{\mathrm{pvi}-\mathrm{pei}}{\mathrm{pei}}\right) * 100\right) / \mathrm{n}\right] ; \mathrm{d}\right)$ varianza del error de predicción $\left.\left[\mathrm{VEP}=\sum_{\mathrm{i}=1}^{\mathrm{n}}(\mathrm{pei}-\mathrm{pvi})^{2} / \mathrm{n}\right] ; \mathrm{e}\right)$ estadístico Durbin Watson [DW=2(1- $\left.\left.) ; \rho=\frac{\sum_{\mathrm{t}=2}^{\mathrm{n}}\left(\mathrm{e}_{\mathrm{t}}-\mathrm{e}_{\mathrm{t}-1}\right)^{2}}{\sum_{\mathrm{t}=1}^{\mathrm{n}} \mathrm{e}_{\mathrm{t}}{ }^{2}}\right] ; \mathrm{f}\right)$ coeficiente de determinación $\left[\mathrm{R}^{2}\right.$ $=(1-(\mathrm{sce} / \mathrm{sct}))] ; \mathrm{y}, \mathrm{g})$ error estándar general o del modelo, a partir de la raíz del cuadrado medio del error $\left(\mathrm{EEG}=\sqrt{\frac{s c e}{n-p-1}}\right.$. Donde: pvi $=$ peso vivo $(\mathrm{kg})$ en la $i-$ ésima edad $(\mathrm{d}) ;$ pei $=$ peso vivo (kg) estimado para la $i$ - ésima edad (d); $\mathrm{n}=$ número total de datos; sce $=$ suma de cuadrados del error; sct = suma de cuadrados total; $\mathrm{k}=$ número de parámetros en el modelo; $1 \mathrm{n}=$ logaritmo natural. El EPP analiza la relación que existe entre el peso medido y el peso estimado, y en función del signo, el MNL sobreestima (+) o subestima (-) las predicciones. Para EPP, VEP, EEG, AIC y BIC, el modelo con el menor valor se consideró como de mejor ajuste, mientras que para $\mathrm{R}^{2}$ fue el mayor valor. El DW analiza las auto correlaciones en los errores, con tres planteamientos: si $2<\mathrm{DW}<4$ existe auto correlación negativa; si $0<\mathrm{DW}<2$ revela ausencia de auto correlación; $\mathrm{y}$, si $\mathrm{DW} \leq 0$ indica que existe auto correlación positiva. 


\section{Resultados y discusión}

El Cuadro 3 muestra los resultados de los criterios estadísticos utilizados para la selección del modelo de mejor ajuste para cada una de las razas. Con base en el $\mathrm{R}^{2}$, todos los MNL explicaron $94 \%$ o más de la variabilidad en la información analizada; además, todos los MNL tienden a subestimar las predicciones (EPP negativo) sin autocorrelación en los residuales $(0<\mathrm{DW}<2)$. Los resultados en la VEP y EEG fueron similares dentro de raza, aunque más altos para el modelo LOG en todas las razas. Con base en AIC y BIC, los modelos MIT y BRO tuvieron resultados similares dentro de raza y fueron los de mejor ajuste para KT, BB, DR y RB; sin embargo, para las razas HS, PE y SF el modelo de mejor ajuste fue el VBE, con EPI entre 40 y $57 \mathrm{~d}$ (Cuadro 4), edad que se ubican en el periodo predestete. Con base en lo MNL que presentan punto de inflexión, el PPI promedio para PE, HS y SF fue de 16.4, 20.2 y $23.2 \mathrm{~kg}$, respectivamente.

Cuadro 3: Estadísticos utilizados para la selección del modelo no lineal de mejor ajuste

\begin{tabular}{rlrrrrrrr}
\hline $\mathbf{R Z}^{\dagger}$ & MOD & ${ }^{\S}$ VEP & ${ }^{*}$ EPP & ${ }^{*} \mathbf{D W}$ & ${ }^{*} \mathbf{R}^{\mathbf{2}}$ & ${ }^{*} \mathbf{E E G}$ & ${ }^{*}$ AIC & ${ }^{*}$ BIC \\
\hline BB & LOG & 20.4 & -17.8 & 0.66 & 0.95 & 4.3 & 55904 & 55927 \\
& GOM & 19.3 & -10.5 & 0.58 & 0.95 & 4.2 & 54563 & 54587 \\
& VBE & 19.1 & -8.4 & 0.56 & 0.95 & 4.1 & 54202 & 54225 \\
& VER & 19.9 & -13.5 & 0.62 & 0.95 & 4.2 & 54942 & 54966 \\
& MIT & 18.8 & -5.9 & 0.54 & 0.95 & 4.1 & 53757 & 53781 \\
& BRO & 19.0 & -6.0 & 0.56 & 0.95 & 4.1 & 53757 & 53781 \\
\hline DR & LOG & 44.3 & -18.4 & 1.30 & 0.95 & 6.4 & 132665 & 132690 \\
& GOM & 41.7 & -10.5 & 1.30 & 0.95 & 6.1 & 130012 & 130037 \\
& VBE & 41.1 & -7.9 & 1.32 & 0.96 & 6.1 & 129282 & 129307 \\
& VER & 42.2 & -9.8 & 1.31 & 0.95 & 6.2 & 130754 & 130779 \\
& MIT & 40.5 & -5.4 & 1.36 & 0.96 & 6.0 & 128389 & 128415 \\
& BRO & 41.0 & -5.8 & 1.39 & 0.96 & 6.0 & 128389 & 128415 \\
\hline HS & LOG & 44.3 & -12.4 & 0.04 & 0.95 & 5.7 & 26115 & 26135 \\
& GOM & 42.8 & -7.3 & 0.04 & 0.95 & 5.6 & 25799 & 25820 \\
& VBE & 42.6 & -6.3 & 0.04 & 0.96 & 5.6 & 25749 & 25770 \\
& VER & 43.7 & -9.9 & 0.04 & 0.95 & 5.6 & 25876 & 25897 \\
& MIT & 42.8 & -5.2 & 0.04 & 0.96 & 5.6 & 25755 & 25775 \\
& BRO & 42.8 & -5.4 & 0.04 & 0.96 & 5.6 & 25755 & 25775 \\
\hline KT & LOG & 37.1 & -17.0 & 0.68 & 0.95 & 6.0 & 262113 & 262141 \\
& GOM & 35.6 & -9.9 & 0.64 & 0.95 & 5.8 & 257855 & 257882 \\
& VBE & 35.3 & -8.0 & 0.64 & 0.95 & 5.8 & 256792 & 256819 \\
& VER & 35.9 & -9.1 & 0.66 & 0.95 & 5.9 & 259020 & 259048 \\
& MIT & 35.3 & -6.1 & 0.68 & 0.95 & 5.7 & 255755 & 255782 \\
& & & & 668 & & & &
\end{tabular}




\begin{tabular}{|c|c|c|c|c|c|c|c|c|}
\hline & $\mathrm{BRO}$ & 35.4 & -6.1 & 0.67 & 0.95 & 5.7 & 255755 & 255782 \\
\hline \multirow[t]{6}{*}{$\mathrm{PE}$} & LOG & 26.4 & -15.1 & 0.26 & 0.94 & 4.6 & 118402 & 118428 \\
\hline & GOM & 25.6 & -9.3 & 0.24 & 0.94 & 4.5 & 116815 & 116841 \\
\hline & VBE & 25.5 & -7.0 & 0.24 & 0.94 & 4.5 & 116583 & 116608 \\
\hline & VER & 26.1 & -8.6 & 0.25 & 0.94 & 4.5 & 117161 & 117187 \\
\hline & MIT & 25.6 & -5.2 & 0.24 & 0.94 & 4.5 & 116745 & 116771 \\
\hline & BRO & 26.2 & -5.9 & 0.26 & 0.94 & 4.5 & 116745 & 116771 \\
\hline \multirow[t]{6}{*}{$\mathrm{RB}$} & LOG & 19.8 & -5.6 & 1.80 & 0.98 & 4.4 & 21873 & 21894 \\
\hline & GOM & 18.7 & -4.9 & 1.80 & 0.98 & 4.2 & 21119 & 21139 \\
\hline & VBE & 18.5 & -4.2 & 1.80 & 0.98 & 4.1 & 20914 & 20935 \\
\hline & VER & 19.1 & -5.1 & 1.81 & 0.98 & 4.0 & 21355 & 21376 \\
\hline & MIT & 18.3 & -3.5 & 1.80 & 0.98 & 4.0 & 20629 & 20650 \\
\hline & BRO & 18.4 & -3.7 & 1.82 & 0.98 & 4.0 & 20629 & 20650 \\
\hline \multirow[t]{6}{*}{$\mathrm{SF}$} & LOG & 46.8 & -9.1 & 0.04 & 0.95 & 6.4 & 37846 & 37867 \\
\hline & GOM & 45.0 & -7.3 & 0.04 & 0.96 & 6.2 & 37354 & 37376 \\
\hline & VBE & 44.8 & -6.1 & 0.06 & 0.96 & 6.2 & 37276 & 37298 \\
\hline & VER & 45.9 & -9.1 & 0.05 & 0.96 & 6.3 & 37467 & 37489 \\
\hline & MIT & 44.8 & -5.3 & 0.06 & 0.96 & 6.2 & 37277 & 37299 \\
\hline & BRO & 44.9 & -5.3 & 0.07 & 0.96 & 6.2 & 37277 & 37299 \\
\hline
\end{tabular}

'Razas: $\mathrm{BB}=$ Blackbelly, $\mathrm{PE}=$ Pelibuey, DR= Dorper, $\mathrm{KT}=$ Katahdin, $\mathrm{SF}=$ Suffolk, HS= Hampshire, $\mathrm{RB}=$ Rambouillet.

${ }^{\S}$ Modelos: VER= Verhulst, LOG= Logístico, VBE= Von Bertalanffy, GOM= Gompertz, BRO= Brody, MIT= Mitscherlich.

*Estadísticos para la selección de modelos: VEP= Varianza del error de predicción, EPP= Error promedio de predicción, DW= Estadístico Durbin-Watson, $\mathrm{R}^{2}=$ Coeficiente de determinación, EEG= Error estándar general, $\mathrm{AIC}=$ Criterio de información Akaike, $\mathrm{BIC}=$ Criterio de información Bayesiano.

Cuadro 4: Coeficientes de regresión e indicadores de crecimiento que conforman los modelos no lineales evaluados

\begin{tabular}{|c|c|c|c|c|c|c|}
\hline $\mathbf{R Z}^{\dagger}$ & MOD $^{\S}$ & ${ }^{¥} \beta_{1} \pm$ ee & ${ }^{\ddagger} \beta_{2} \pm$ ee & ${ }^{¥} \beta_{3} \pm$ ee & ${ }^{\mathfrak{E}} \mathbf{P P I}$ & ${ }^{£} \mathbf{E P I}$ \\
\hline \multirow[t]{6}{*}{$\mathrm{BB}$} & LOG & $33.1 \pm 0.13$ & $7.37 \pm 0.08$ & $0.0243 \pm 0.0001$ & 16.6 & 82 \\
\hline & GOM & $36.9 \pm 0.21$ & $2.42 \pm 0.01$ & $0.0139 \pm 0.0001$ & 13.6 & 63 \\
\hline & VBE & $40.0 \pm 0.28$ & $0.575 \pm 0.01$ & $0.0103 \pm 0.0001$ & 11.9 & 53 \\
\hline & VER & $35.2 \pm 0.17$ & $3.35 \pm 0.02$ & $0.0171 \pm 0.0002$ & 17.6 & 86 \\
\hline & MIT & $61.3 \pm 1.22$ & $-13.11 \pm 0.03$ & $0.0034 \pm 0.0002$ & & \\
\hline & BRO & $61.2 \pm 1.31$ & $0.955 \pm 0.02$ & $0.0034 \pm 0.0002$ & & \\
\hline \multirow[t]{5}{*}{ DR } & LOG & $48.8 \pm 0.14$ & $7.61 \pm 0.07$ & $0.0242 \pm 0.0001$ & 24.4 & 84 \\
\hline & GOM & $54.1 \pm 0.22$ & $2.48 \pm 0.01$ & $0.0140 \pm 0.0001$ & 19.9 & 65 \\
\hline & VBE & $58.6 \pm 0.29$ & $0.586 \pm 0.01$ & $0.0105 \pm 0.0001$ & 17.4 & 54 \\
\hline & VER & $51.8 \pm 0.17$ & $3.43 \pm 0.01$ & $0.0171 \pm 0.0001$ & 25.9 & 88 \\
\hline & & & 669 & & & \\
\hline
\end{tabular}




\begin{tabular}{|c|c|c|c|c|c|c|}
\hline & MIT & $88.8 \pm 1.23$ & $-11.52 \pm 0.22$ & $0.0035 \pm 0.0001$ & & \\
\hline & $\mathrm{BRO}$ & $88.9 \pm 1.22$ & $0.959 \pm 0.0$ & $0.0036 \pm 0.0001$ & & \\
\hline \multirow[t]{6}{*}{$\mathrm{HS}$} & $\mathrm{LOG}$ & $45.4 \pm 0.24$ & $6.99 \pm 0.13$ & $0.0285 \pm 0.0003$ & 22.7 & 68 \\
\hline & GOM & $50.0 \pm 0.37$ & $2.31 \pm 0.02$ & $0.0163 \pm 0.0002$ & 18.3 & 51 \\
\hline & VBE & $53.2 \pm 0.48$ & $0.552 \pm 0.03$ & $0.0125 \pm 0.0001$ & 15.8 & 40 \\
\hline & VER & $48.0 \pm 0.31$ & $3.22 \pm 0.03$ & $0.0201 \pm 0.0002$ & 24.0 & 71 \\
\hline & MIT & $68.6 \pm 1.36$ & $-12.31 \pm 0.49$ & $0.0054 \pm 0.0002$ & & \\
\hline & BRO & $68.6 \pm 1.36$ & $0.935 \pm 0.01$ & $0.0054 \pm 0.0001$ & & \\
\hline \multirow[t]{6}{*}{$\mathrm{KT}$} & LOG & $43.5 \pm 0.09$ & $7.42 \pm 0.04$ & $0.0241 \pm 0.0001$ & 21.8 & 83 \\
\hline & GOM & $48.6 \pm 0.15$ & $2.44 \pm 0.01$ & $0.0138 \pm 0.0001$ & 17.9 & 65 \\
\hline & VBE & $52.9 \pm 0.21$ & $0.581 \pm 0.01$ & $0.0102 \pm 0.0001$ & 15.7 & 54 \\
\hline & VER & $46.3 \pm 0.12$ & $3.38 \pm 0.01$ & $0.0171 \pm 0.0002$ & 23.2 & 86 \\
\hline & MIT & $84.9 \pm 1.01$ & $-12.83 \pm 0.17$ & $0.0032 \pm 0.0001$ & & \\
\hline & BRO & $84.9 \pm 0.98$ & $0.959 \pm 0.01$ & $0.0032 \pm 0.0001$ & & \\
\hline \multirow[t]{6}{*}{$\mathrm{PE}$} & LOG & $35.9 \pm 0.01$ & $8.48 \pm 0.07$ & $0.0256 \pm 0.0001$ & 17.9 & 83 \\
\hline & GOM & $40.7 \pm 0.18$ & $2.57 \pm 0.01$ & $0.0141 \pm 0.0001$ & 14.9 & 67 \\
\hline & VBE & $44.7 \pm 0.24$ & $0.597 \pm 0.01$ & $0.0102 \pm 0.0001$ & 13.2 & 57 \\
\hline & VER & $38.7 \pm 0.14$ & $3.55 \pm 0.02$ & $0.0174 \pm 0.0001$ & 19.4 & 88 \\
\hline & MIT & $78.9 \pm 1.40$ & $-12.01 \pm 0.22$ & $0.0029 \pm 0.0001$ & & \\
\hline & $\mathrm{BRO}$ & $78.9 \pm 1.41$ & $0.966 \pm 0.01$ & $0.0029 \pm 0.0001$ & & \\
\hline \multirow[t]{6}{*}{$\mathrm{RB}$} & LOG & $42.7 \pm 0.16$ & $6.05 \pm 0.09$ & $0.0259 \pm 0.0002$ & 21.4 & 69 \\
\hline & GOM & $45.9 \pm 0.23$ & $2.14 \pm 0.02$ & $0.0157 \pm 0.0001$ & 16.9 & 48 \\
\hline & VBE & $48.1 \pm 0.28$ & $0.524 \pm 0.02$ & $0.0124 \pm 0.0001$ & 14.3 & 36 \\
\hline & VER & $44.5 \pm 0.19$ & $3.00 \pm 0.02$ & $0.0191 \pm 0.0001$ & 22.3 & 70 \\
\hline & MIT & $56.8 \pm 0.62$ & $-13.96 \pm 0.32$ & $0.0064 \pm 0.0001$ & & \\
\hline & BRO & $56.9 \pm 0.61$ & $0.915 \pm 0.01$ & $0.0064 \pm 0.0001$ & & \\
\hline \multirow[t]{6}{*}{$\mathrm{SF}$} & LOG & $51.7 \pm 0.23$ & $7.67 \pm 0.13$ & $0.0276 \pm 0.0002$ & 25.8 & 74 \\
\hline & GOM & $57.5 \pm 0.36$ & $2.42 \pm 0.02$ & $0.0155 \pm 0.0001$ & 21.1 & 57 \\
\hline & VBE & $61.6 \pm 0.49$ & $0.571 \pm 0.01$ & $0.0117 \pm 0.0001$ & 18.3 & 46 \\
\hline & VER & $55.1 \pm 0.36$ & $3.38 \pm 0.02$ & $0.0191 \pm 0.0001$ & 27.6 & 77 \\
\hline & MIT & $84.0 \pm 1.61$ & $-12.03 \pm 0.35$ & $0.0046 \pm 0.0001$ & & \\
\hline & BRO & $84.0 \pm 1.61$ & $0.945 \pm 0.01$ & $0.0046 \pm 0.0001$ & & \\
\hline
\end{tabular}

${ }^{\dagger}$ Razas: BB= Blackbelly, PE= Pelibuey, DR= Dorper, KT= Katahdin, SF= Suffolk, HS= Hampshire, RB= Rambouillet.

${ }^{\S}$ Modelos: VER= Verhulst, LOG= Logístico, VBE= Von Bertalanffy, GOM= Gompertz, BRO= Brody, MIT= Mitscherlich.

${ }^{¥}$ Coeficientes de regresión que conforman los modelos no lineales: $\beta_{1=}$ valor asintótico $(\mathrm{kg}), \beta_{2}=$ parámetro de ajuste, $\beta_{3}=$ tasa de crecimiento, ee $=$ error estándar.

${ }^{\mathfrak{f}}$ Indicadores de crecimiento: EPI= edad (días) al punto de inflexión, PPI= peso (kg) al punto de inflexión.

Las curvas de crecimiento, con base en el modelo de mejor ajuste, mostraron las diferencias en el patrón de crecimiento a través de razas (Figuras 1 y 2). La curva de crecimiento describe y 
representa la evolución del peso vivo a través del tiempo; el análisis de las curvas de crecimiento proporciona información que puede ser utilizada en los programas de manejo, alimentación y mejora genética. Los MNL expresan la curva de crecimiento en función de varios componentes: peso adulto, tasa o velocidad de crecimiento, grado de madurez, edad y el peso al punto de inflexión, entre otros ${ }^{(2,7)}$; por consiguiente, para modificar o alterar el crecimiento se deben buscar estrategias que trasciendan en los citados componentes ${ }^{(14,15)}$. El modelo VBE se caracteriza por una curva sigmoide (Figura 2), siendo el punto de inflexión donde la TAC cambia de un proceso de aceleración a una fase de desaceleración; mientras que los modelos BRO y MIT describen una curva de crecimiento continua y sin punto de inflexión (Figura 1), y la TAC como proporción del PAD es constante a través del tiempo ${ }^{(3,16)}$.

Figura 1: Curvas de crecimiento para borregas de las razas Katahdin (KT), Blackbelly (BB), Dorper (DR) y Rambouillet (RB), con base en el modelo de Brody

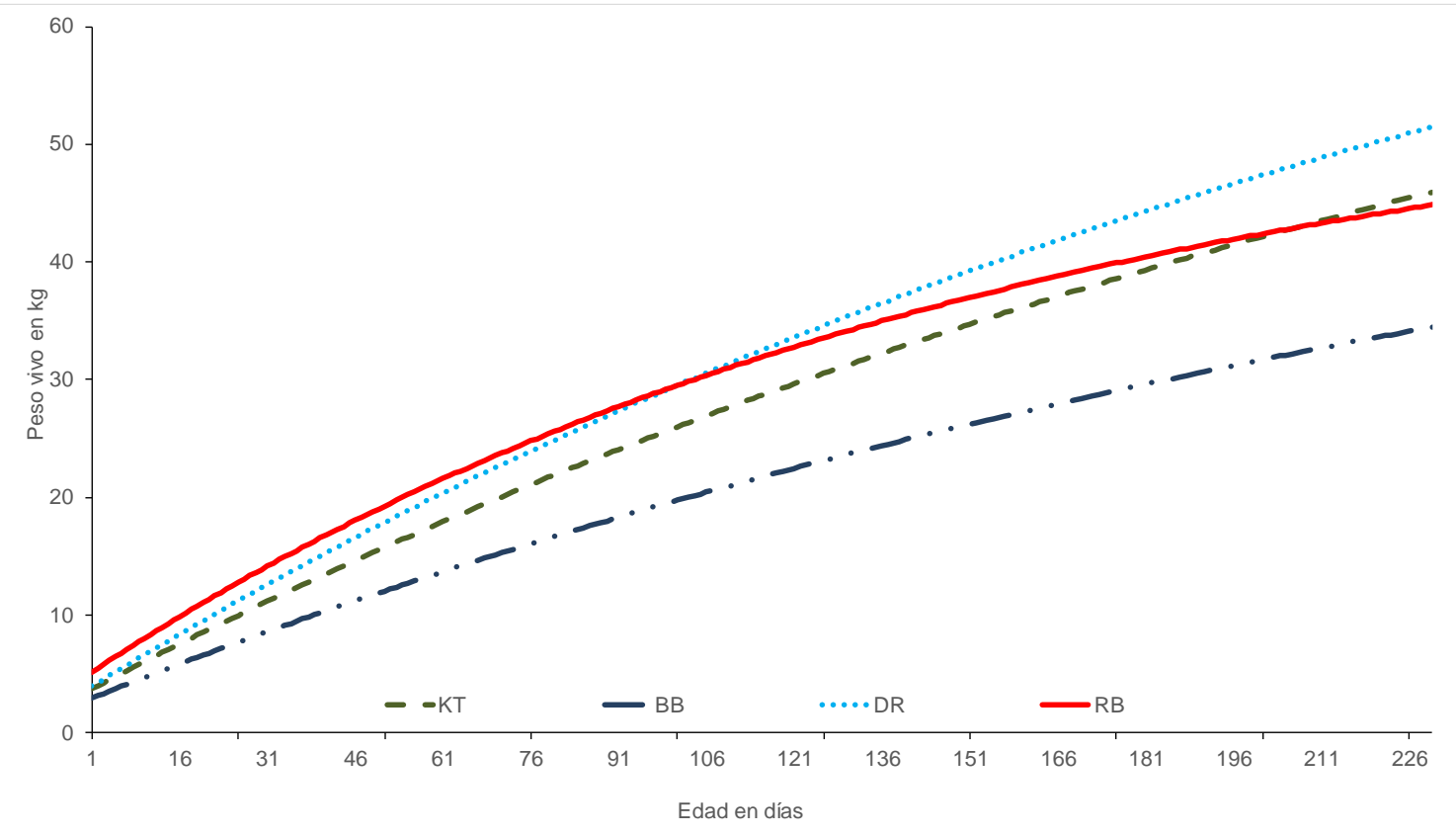

En estudios similares, Bahreini $e t a l^{(5)}$ en ovinos Baluchi, Kopuzlu et al $l^{(17)}$ en borregas Hemsin, y Gbangboche et $a l^{(18)}$ en ovinos West African Dwarf reportaron que el modelo BRO fue de mejor ajuste para describir el crecimiento; así mismo, analizando el crecimiento de ovinos Morada Nova $^{(4)}$, publicaron que los modelos Meloun I y Meloun III, con patrones de crecimiento similares a los modelos BRO y MIT del presente estudio, fueron los de mejor ajuste. Por otro lado, Lupi $e t a l^{(9)}$ en borregas Segureñas y Topal $e t a l^{(19)}$ en ovinos Awassi, reportaron que el modelo VBE fue el de mejor ajuste. 
Figura 2: Curvas de crecimiento para borregas de las razas Pelibuey (PE), Suffolk (SF) y Hampshire (HS), con base en el modelo Von Bertalanffy

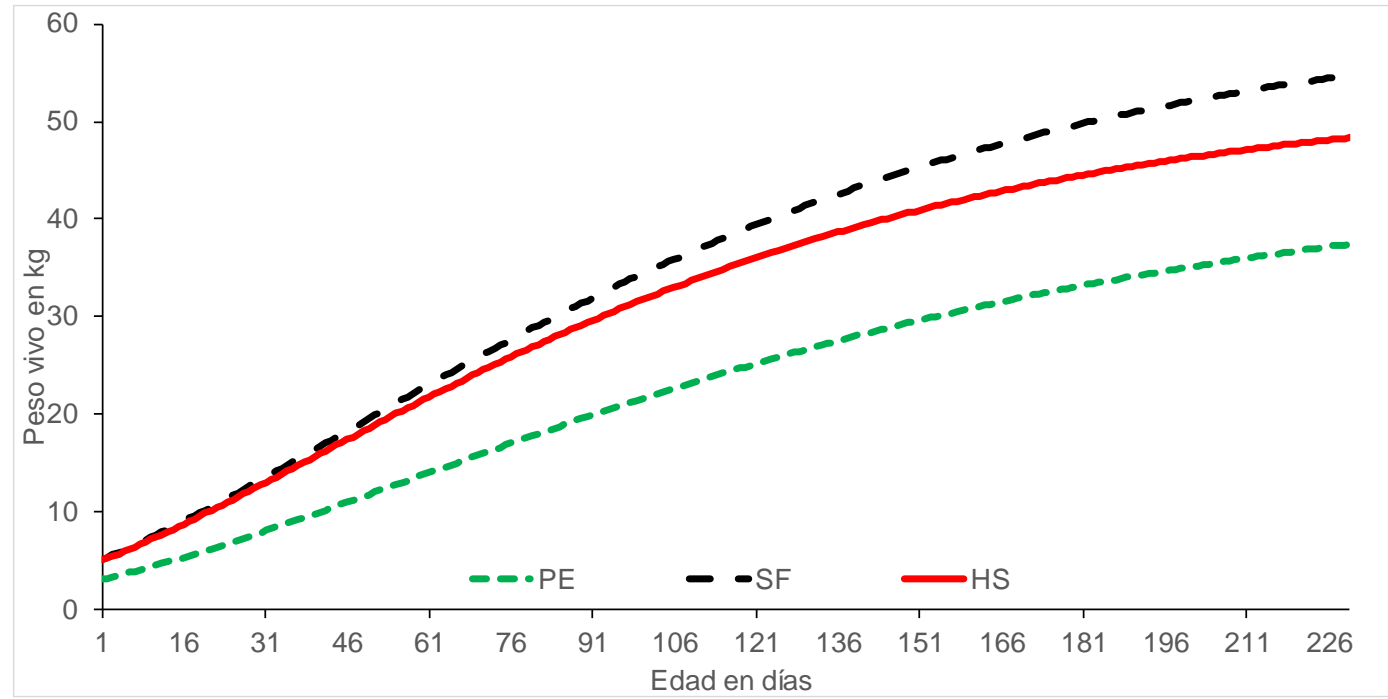

Para el PAD (Cuadro 4) se observaron marcadas diferencias a través de las razas evaluadas, con la tendencia del más alto para los modelos BRO y MIT y el menor para LOG y VER; con valores promedio de $44.6 \mathrm{~kg}$ en BB, 49.2 kg en RB, 52.9 kg en PE, 55.6 kg en HS, $60.2 \mathrm{~kg}$ en KT, 64.7 en $\mathrm{SF}$ y 65.2 en DR. Incrementos del PAD en las hembras repercute en las necesidades de mantenimiento, reproducción y valor de desecho; en la borrega se ejerce gran porcentaje de los gastos para la producción de un cordero, aumentar el tamaño de las hembras repercute en el aumento de los costos de producción; sin embargo, en los programas de selección se puede mantener constante el peso asintótico, mientras se maximiza la $\mathrm{TAC}^{(14,20)}$. La TAC se refiere a la rapidez de crecimiento relativo al PAD; con TAC altas se alcanza el PAD a menor edad. La velocidad de crecimiento es económicamente importante porque se puede usar para determinar el momento óptimo del sacrificio, una vez que el animal haya alcanzado la velocidad de crecimiento máxima $^{(13,21)}$.

Las correlaciones entre PAD y TAC son esenciales en las estrategias para modificar las curvas de crecimiento $^{(15,21)}$. En el presente estudio todas las correlaciones entre PAD y TAC fueron negativas y altas (-0.70 a -0.99). La correlación negativa puede indicar ciertas características de las curvas de crecimiento: a) los mayores PAD no derivan de altas TAC; b) una menor TAC puede incrementar el tiempo para alcanzar el PAD; y c) en los esquemas de mejoramiento genético, se puede incrementar la TAC sin repercusiones en el $\operatorname{PAD}^{(7,15,22)}$. 


\section{Conclusiones e implicaciones}

Para las razas Hampshire, Pelibuey y Suffolk, con base en el modelo de Von Bertalanffy, la curva de crecimiento fue de tipo sigmoidea, con un punto de inflexión en los intervalos de 40 a 57 días. Para Katahdin, Blackbelly, Dorper y Rambouillet, la curva de crecimiento presentó una tasa de crecimiento continua y sin punto de inflexión, dado las características del modelo de Brody. Las diferencias a través de razas, dado el patrón de la curva y los indicadores de crecimiento, expresan un potencial genético que puede ser favorable en los diversos sistemas de producción.

\section{Agradecimientos}

Se agradece al Organismo de la Unidad Nacional de Ovinocultores por facilitar la base de datos para realizar el presente estudio, en el marco del convenio de colaboración entre la Universidad Autónoma de Chihuahua y el Consejo Nacional de los Recursos Genéticos Pecuarios. Se agradece al Consejo Nacional de Ciencia y Tecnología por el apoyo económico otorgado al segundo autor para realizar estudios de maestría en ciencias.

\section{Literatura citada:}

1. CONARGEN. Guía técnica de programas de control de producción y mejoramiento genético en ovinos. Consejo Nacional de los Recursos Genéticos Pecuarios. México, DF. 2010.

2. Lewis RM, Emmans GC, Dingwall WS, Simm G. A description of the growth of sheep and its genetic analysis. Anim Sci 2002;74:51-62.

3. Agudelo GDA, Cerón MF, Restrepo LFB. Modelación de las funciones de crecimiento aplicadas a la producción animal. Rev Colomb Cienc Pecu 2008;21:39-58.

4. de Andrade SL, Souza PLC, Mendes CHM, Fonseca S, Gomes da SF. Traditional and alternative nonlinear models for estimating the growth of Morada Nova sheep. Rev Bras Zootec 2013;42:651-655.

5. Bahreini BMR, Aslaminejad AA, Sharifi AR, Simianer H. Comparison of mathematical models for describing the growth of Baluchi sheep. J Agr Sci Tech 2014;14:57-68.

6. Teixeira NMR, da Cruz JF, Neves FH, Santos SE, Souza CPL, Mendes MC. Descrição do crescimento de ovinos Santa Inês utilizando modelos não-lineares seleccionados por análise multivariada. Rev Bras Saude Prod Anim 2016;17:26-36. 
7. Malhado CHM, Carneiro PL, Alfonso PRA, Souza AA, Sarmento. Growth curves in Dorper sheep crossed with the local Brazilian breeds, Morada Nova, Rabo Largo, and Santa Inês. Small Ruminant Res 2009;84:16-21.

8. Ben HM, Atti N. Comparison of growth curves of lamb fat tail measurements and their relationship with body weight in Babarine sheep. Small Ruminant Res 2013;95:120-127.

9. Lupi TM, Nogales S, León JM, Barba C, Delgado JV. Characterization of commercial and biological growth curves in the Segureña sheep breed. Animal 2015;9:1341-1348.

10. SAS. SAS/STAT User's Guide (Release 9.0). Cary NC, USA: SAS Inst. Inc. 2005.

11. Motulsky H, Christopoulos A. Fitting models to biological data using linear and nonlinear regression. A practical guide to curve fitting. Graph Pad Software Inc. 2003.

12. Hossein-Zadeh NG. Modeling the growth curve of Iranian Shall sheep using non-linear growth models. Small Ruminant Res 2015;130:60-66.

13. Hossein-Zadeh NG, Golshani M. Comparison of non-linear models to describe growth of Iranian Guilan sheep. Rev Colomb Cienc Pecu 2016;29:199-209.

14. Owens FN, Dubeski P, Hanson CF. Factors that alter the growth and development of ruminants. J Anim Sci 1993;71:3138-3150.

15. Lupi TM, León JM, Nogales S, Barba C, Delgado JV. Genetic parameters of traits associated with the growth curve in Segureña sheep. Animal 2016;9:729-735.

16. Ribeiro de FA. Curvas de crescimento na produçã animal. Rev Bras Zootec 2005;34:786-795.

17. Kopuzlu S, Sezgin E, Esenbuga E, Bilgin OC. Estimation of growth curve characteristics of Hemsin male and female sheep. J Appl Anim Res 2014;42:228-232.

18. Gbangboche AB, Glele-Kakai R, Salifou S, Albuquerque LG, Leroy PL. Comparison of nonlinear growth models to describe the growth curve in West African Dwarf sheep. Animal 2008;2:1003-1012.

19. Topal M, Ozdemir M, Aksakal V, Yildiz N, Dogru U. Determination of the best nonlinear function in order to estimate growth in Morkaraman and Awassi lambs. Small Ruminant Res 2004;55:229-232. 
20. Bathaei SS, Leroy PL. Growth and mature weight of Mehraban Iranian fat-tailed sheep. Small Ruminant Res 1996;22:155-162.

21. Lambe NR, Navajas EA, Simm G, Bünger L. A genetic investigation of various growth models to describe growth of lamb of two contrasting breeds. J Anim Sci 2006;84:2642-2654.

22. Acioli da SLS, Bossi FA, de Lima da SF, Mendes GB, de Oliveira SR, Tonhati H, da Costa BC. Growth curve in Santa Inês sheep. Small Ruminant Res 2012;105:182-185. 